\title{
ESTILO DE VIDA EN ESTUDIANTES DE MEDICINA EN INTERNADO ROTATORIO DEL HOSPITAL MARIO CATARINO RIVAS.
}

\section{LIFESTYLE OF MEDICAL STUDENTS IN ROTATING INTERNSHIP OF MARIO CATARINO RIVAS HOSPITAL.}

*Myriam Elizabeth Vijil Mejía, *Mario René Valeriano Lanza, **Bismark Espinoza Espinal.

\section{RESUMEN}

El estilo de vida tiene una influencia significativa en la salud física y mental de los seres humanos y por ende forma parte de los objetivos de desarrollo sostenible promovidos por las Naciones Unidas. Objetivo: Caracterizar el estilo de vida de los estudiantes de internado rotatorio según sexo y rotación cursada de la carrera de medicina en el Hospital Mario Catarino Rivas (HMCR) en julio del 2017. Pacientes y métodos: Estudio cuantitativo, transversal y descriptivo. La población fue de 275 estudiantes matriculados en internado rotatorio en el HMCR y la muestra 118. Se utilizó el cuestionario de Perfil de Estilo de Vida de Nola Pender Resultados: Edad media de 24 años, $52.5 \%$ eran hombres y $47.5 \%$ mujeres, de los cuales $31.4 \%$ alterno por medicina interna, $28.8 \%$ por cirugía, $23.7 \%$ por pediatría y $16.1 \%$ por ginecología. Predominó un estilo de vida medio en el $50.0 \%$ de los internos, para ambos sexos. Se observó la prevalencia de un estilo de vida alto en $52.6 \%$ de los internos cursando ginecología y $42.9 \%$ en aquellos cursando pediatría al momento del estudio. Las rotaciones de cirugía y medicina interna reportaron un predominio de estilo de vida medio, de $55.9 \%$ y $59.5 \%$, respectivamente. Conclusiones: De manera global, en el $50 \%$ de la población predominó un estilo de vida medio. El género no influyó sobre el estilo de vida de los internos, pero la rotación cursada sí presentó diferencias: en ginecología y pediatría dominó un estilo de vida alto.

\footnotetext{
*Estudiante de VII año de la carrera de medicina en la Escuela Universitaria de Ciencias de la Salud de la Universidad Nacional Autónoma de Honduras en el Valle de Sula (EUCS/UNAH-VS)

mariovaleriano25@gmail.com https://orcid.org/0000-0003-3251-9205 myriamvijilm@gmail.com https://orcid.org /0000-0003-2029-5320

** Médico Psiquiatra, jefe del departamento de Psiquiatría en Hospital Mario Catarino Rivas

gabrielesp766@gmail.com https://orcid.org/0000-0003-2812-1050 Recibido: 15 de mayo 2018 Aprobado: 30 de noviembre 2018
}

\section{PALABRAS CLAVE}

Estilo de vida, Estudiantes del área de la salud Honduras, Internado.

\section{ABSTRACT}

Lifestyle has a significant influence on the physical and mental health of human beings hence it is part of the sustainable development objectives promoted by the United Nations. Objective: To characterize the lifestyle of medical interns of the Hospital Mario Catarino Rivas (HMCR) in July 2017, comparing by sex and rotation coursed. Researech Methodology: A quantitative, cross-sectional and descriptive study was carried out using the Nola Pender Lifestyle Profile Questionnaire. The study population consisted of students enrolled in rotating internships at the MCRH at the time of the study (sample $=275$, sample $=118$ ). Results: The average age is 24 years old, $52.5 \%$ of the population were men and $47.5 \%$ women. $31.4 \%$ rotated by Internal Medicine, $28.8 \%$ by surgery, $23.7 \%$ by Pediatrics and $16.1 \%$ by Gynecology. The interna, both gender, manifested an average lifestyle of $50.0 \%$. The prevalence of a high lifestyle was observed in $52.6 \%$ of the Gynecology interns and in $42.9 \%$ of those studying Pediatrics at the study time. The rotations of Surgery and Internal Medicine reported a predominance of average lifestyle, of $55.9 \%$ and $59.5 \%$, respectively. Conclusions: Overall and average lifestyle predominated in $50 \%$ : of the population without the gender influence in the medical interns. Lifestyle even though the rotation carried, out some differences as a high lifestyle prevailed in ginecology and pediatrics.

\section{KEYWORDS}

Life style, Students Heath ocuppations Honduras, Intern ship and residency. 


\section{INTRODUCCIÓN}

En 1946 la Organización Mundial de la Salud definió en su constitución el concepto de salud como "un estado de completo bienestar físico, mental y social, y no solamente la ausencia de afecciones o enfermedades". ${ }^{(1)}$ Esta definición ofrece una perspectiva de la salud general, que debe incluir todos los aspectos de la vida de las personas: físico, mental, social, aspectos ambientales y económicos asi cómo la manera en la que la interacción de estos factores determinan el bienestar de los individuos. ${ }^{(2)}$ Nola Pender introdujo en 1996 un instrumento de medición de estilo de vida que contempla seis dimensiones: nutrición, ejercicio, responsabilidad en salud, manejo del estrés, relaciones interpersonales y auto actualización, integrándolas en un cuestionario que fué el instrumento de esta investigación.

El estilo de vida se define como el conjunto de pautas y hábitos comportamentales cotidianos de una persona, que demuestran cierta consistencia en el tiempo, bajo condiciones más o menos constantes y que pueden constituirse en dimensiones de riesgo o de seguridad dependiendo de su naturaleza. (3) Se considera un proceso social que involucra una serie de tradiciones, hábitos y conductas de los individuos y grupos, que conllevan a la satisfacción de necesidades humanas para alcanzar el bienestar y una vida más satisfactoria. ${ }^{(4)}$

El estilo de vida tiene una influencia significativa en la salud física y mental de los seres humanos ${ }^{(5)}$ y por ende forma parte de los objetivos de desarrollo sostenible promovidos por las Naciones Unidas. ${ }^{(6)}$

Los comportamientos individuales de estilo de vida se encuentran entre los cinco determinantes múltiples de la salud tal como lo define Healthy People 2020, los objetivos nacionales a 10 años basados en la ciencia para mejorar la salud de todos los estadounidenses. Los otros cuatro determinantes son medio ambiente social, cuidado de la salud, genética y biología.(7)
Durante la Conferencia Mundial de Promoción de la Salud en Shanghai 2016 se elaboraron 12 consejos para gozar de buena salud, entre los cuales figuraron seguir una dieta saludable y gestionar el estrés para una mejor salud física y mental. ${ }^{(8)}$ La nutrición implica la selección, conocimiento y consumo de alimentos esenciales para el sustento, la salud y el bienestar. ${ }^{(9)}$ Diversos estudios ponen de manifiesto la influencia que tiene el desayuno con el rendimiento físico e intelectual en las actividades que se realizan durante la mañana y es considerado como una de las comidas más importantes en la dieta de un individuo. ${ }^{(10)}$

La actividad física se refiere a todo movimiento del cuerpo que hace trabajar a los músculos y requiere más energía que estar en reposo como caminar, correr, bailar, nadar o practicar yoga. ${ }^{(11)}$ El ejercicio físico regular y realizado de forma adecuada nos ayuda a mantener un peso estable así como mejora el estado del cuerpo y de la mente; ayudándonos a mantener la función cardiovascular, musculo esquelética y metabólica. ${ }^{(3)}$ Jiménez y colaboradores demostraron que las personas que realizan regularmente ejercicio físico se perciben más saludables, con menor estrés y presentan mayor estado de ánimo que aquellas que no realizan ningún tipo de ejercicio físico. ${ }^{(12)}$ En Honduras, el Poder Ejecutivo elaboró y dió a conocer el Decreto No PCM-030-2015 publicado en la Gaceta; donde prioriza la actividad física regular y la alimentación saludable para que se aplique con carácter obligatorio en todas las instituciones que forman parte del aparato estatal en el marco de la jornada diaria laboral. ${ }^{(13)}$ La responsabilidad en salud implica un sentido activo de responsabilidad por el propio bienestar, la educación de uno mismo respecto a su propia salud, incluye la búsqueda de información profesional necesaria. ${ }^{(9)}$

Otra de las dimensiones a estudiar son las relaciones interpersonales manejo del estrés y autorealización incluido en el estilo de vida que va implícito en lo que es la inteli- 
gencia emocional, Bar-On, la define como un conjunto de conocimientos y habilidades en lo emocional y social que influyen en nuestra capacidad general para afrontar efectivamente las demandas de nuestro medio. Su modelo contiene 10 factores principales, entre los cuales figuran la autoconsideración (percepción exacta de uno mismo), relaciones interpersonales, tolerancia al estrés y flexibilidad, ${ }^{(14)}$ evaluados en el cuestionario de Perfil de Estilo de Vida (PEPS-I) de Nola Pender que se aplica en este estudio. Además incluye cinco facilitadores de dichos factores, entre los cuales se menciona la autoactualización y el optimismo, ${ }^{(14)}$ que también se contemplan en el cuestionario.

El manejo del estrés implica la identificación y movilización de recursos psicológicos y físicos para controlar o reducir la tensión de manera efectiva. ${ }^{(9)}$ Calsin-Quinto y Quispe-Ticona concluyeron que llevar buenos hábitos de vida contribuye al bienestar psicólogico de las personas y reduce el estrés en el área de trabajo. ${ }^{(15)} \mathrm{Si}$ bien los médicos internos son aún estudiantes de medicina, su compromiso con el quehacer laboral es equiparable con el de un profesional médico, por tanto, se encuentran expuestos al estrés laboral y al desgaste que de este se derive. ${ }^{(16)}$

Las relaciones interpersonales implican el intercambio de pensamientos y sentimientos a través de mensajes verbales y no verbales para lograr un sentido de intimidad y cercanía dentro de relaciones significativas, en lugar de más informales, con los demás. ${ }^{(9)}$ Finalmente, la auto actualización se refiere a la conciencia de capacidades para la toma de decisiones, reconocimiento de las habilidades que se poseen para el autocuidado y la actitud que se tiene hacia mejorarlas y actualización sobre la información necesaria para el autocuidado de la salud.(9)

El desempeño laboral está relacionado con el estilo de vida de los trabajadores, por lo que le corresponde a quien gestiona, geren- cia o administra una organización averiguar percepciones sobre aspectos que configuren una visión integral, tales como: estilo de vida saludable o poco saludable, quererse a sí mismo, sentirse feliz/contento, miras hacia el futuro, conciencia de lo que importa en la vida, respeto de propios éxitos, propósito de vida, metas realistas, conocimiento, reacciones y estabilidad físico mental.(6) Lo que motivó a los autores a plantearse el siguiente objetivo: caracterizar el estilo de vida de los estudiantes de internado rotatorio comparando según sexo y rotación cursada de la carrera de medicina en el Hospital Mario Catarino Rivas (HMCR) en Julio del 2017.

\section{PACIENTES Y MÉTODOS}

Estudio cuantitativo, transversal y descriptivo, cuya población estudiada consistió en los estudiantes matriculados en el internado rotatorio de la Universidad Nacional Autónoma de Honduras (UNAH) y Universidad Católica de Honduras (UNICAH) que aceptó participar y que se encontraban cursado una rotación en el HMCR al momento del estudio población: 275 / muestra 118.

Criterios de inclusión:

1. Estudiante matriculado en el internado rotatorio, asignado en el HMCR al momento del estudio (julio 2017), de UNAH o UNICAH.

2. Que acepte voluntariamente participar del estudio.

Criterios de exclusión:

1. Estudiantes que no estaban matriculados en el internado rotatorio, no asignados en el HMCR.

2. Que no estuvieran dispuestos a ser parte del estudio.

El menor porcentaje de internos cursando la rotación de Ginecología es porque se envía un tercio de los estudiantes al Hospital Leonardo Martínez Valenzuela por lo que se explica el menor número de internos encuestados en ginecología en comparación con las demás rotaciones (aproximado de 33 encuestados por rotación). 
Se solicitó su consentimiento informado antes de llenar el cuestionario. Finalmente, una vez recolectada la información, se realizó una base de datos y los gráficos correspondientes utilizando el software Numbers 4.1.1 (equivalente al programa Microsoft Excel en el sistema operativo de Windows).

Se utilizó como instrumento el cuestionario de perfil de Estilo de Vida (PEPS-I) creado por Nola Pender y modificado por los autores para adaptar los datos generales a las variables de interés en la población de estudiantes de internado rotatorio. Dicho cuestionario es integrado por 48 reactivos tipo Likert, los cuales se subdividen en las dimensiones de nutrición (6 reactivos), ejercicio (5 reactivos), responsabilidad en salud (10 reactivos), manejo del estrés (7 reactivos), soporte interpersonal (7 reactivos) y auto actualización (13 reactivos).

Dicho cuestionario solicita al encuestado que identifique la frecuencia con la que ha practicado cada enunciado en los últimos 30 días y que circule la respuesta que refleje mejor su forma de vivir actual. Cada reactivo tiene una puntuación mínima de 1 ("nunca") y máxima de 4 ("siempre"), sumando un puntaje global mínimo de 48 y máximo de 192. Las puntuaciones definen estilos de vida alto (132-192), medio (108-131) y bajo
(48-107), según 0.75 de la desviación estándar por arriba o por debajo del puntaje medio.(17)

\section{RESULTADOS}

Los médicos internos tenían una edad mínima de 23 años y máxima de 30 años con una media de 24 años. El $52.5 \%$ (62) fueron hombres y $47.5 \%$ (56) mujeres. El $96.6 \%$ (114) eran solteros, $2.5 \%$ casados (3) y 0.9 (1)\% en unión libre. El $56.4 \%$ (67) residían en San Pedro Sula y $43.6 \%$ (51) procedían de zonas fuera de la ciudad. El $31.4 \%$ cursaban el internado en medicina interna, $28.8 \%$ en cirugía, $23.7 \%$ en pediatría y $16.1 \%$ en ginecología. De manera global el $34.75 \%$ de la población en cuestión resultó llevar un estilo de vida alto, mientras que el $50 \%$ medio y el restante $15.25 \%$ bajo.

El puntaje máximo obtenido fue de 168 en las mujeres y 167 en los hombres, con una media de 124 en ambos sexos y una desviación estándar de 20.86 puntos en mujeres y 18.79 puntos en hombres.

El puntaje máximo posible en cada dimensión evaluada por el cuestionario, el puntaje mínimo y máximo obtenido por sexo, así como la mediana, el promedio y la desviación estándar según cada sexo se muestra en la Tabla No.1.

Tabla No.1: Puntaje Fraccionado en el Cuestionario Perfil de Estilo de Vida de Pender.

\begin{tabular}{|c|c|c|c|c|c|c|c|c|c|c|c|}
\hline \multirow{2}{*}{ Dimensión } & \multirow{2}{*}{$\begin{array}{c}\text { Puntaje } \\
\text { máximo } \\
\text { posible }\end{array}$} & \multicolumn{2}{|c|}{ Mínimo } & \multicolumn{2}{|c|}{ Máximo } & \multicolumn{2}{|c|}{ Mediana } & \multicolumn{3}{|c|}{ Media } & \multicolumn{2}{|c|}{$\begin{array}{c}\text { Desviación } \\
\text { Estándar }\end{array}$} \\
\cline { 4 - 12 } & $\mathrm{M}$ & $\mathrm{H}$ & $\mathrm{M}$ & $\mathrm{H}$ & $\mathrm{M}$ & $\mathrm{H}$ & $\mathrm{M}$ & $\mathrm{H}$ & $\mathrm{M}$ & $\mathrm{H}$ \\
\hline Nutrición & 24 & 6 & 7 & 23 & 24 & 14 & 13 & 13.25 & 13.04 & 3.76 & 3.25 \\
\hline Ejercicio & 20 & 5 & 5 & 17 & 15 & 6 & 8 & 7.02 & 8.49 & 2.47 & 3.0 \\
\hline $\begin{array}{c}\text { Responsa- } \\
\text { bilidad en } \\
\text { Salud }\end{array}$ & $\mathbf{4 0}$ & 12 & 11 & 37 & 30 & 21 & 21 & 21.83 & 20.60 & 6.49 & 5.07 \\
\hline
\end{tabular}




\begin{tabular}{|c|c|c|c|c|c|c|c|c|c|c|c|}
\hline $\begin{array}{c}\text { Manejo } \\
\text { del Estrés }\end{array}$ & $\mathbf{2 8}$ & 8 & 10 & 28 & 28 & 15 & 16 & 15.63 & 16.88 & 4.71 & 4.66 \\
\hline $\begin{array}{c}\text { Soporte } \\
\text { Interper- } \\
\text { sonal }\end{array}$ & 28 & 8 & 10 & 28 & 28 & 22 & 19 & 21.54 & 20 & 5.17 & 4.5 \\
\hline $\begin{array}{c}\text { Autoactua- } \\
\text { lización }\end{array}$ & 52 & 13 & 22 & 52 & 52 & 47 & 47 & 45.25 & 45.26 & 7.60 & 7.62 \\
\hline Global & 192 & 73 & 75 & 168 & 167 & 121 & 125 & 124.53 & 124.28 & 20.86 & 18.79 \\
\hline
\end{tabular}

Fuente: Cuestionario de Perfil de Estilo de Vida (PEPS-I) creado por Nola Pender.

En el puntaje por sexo, se encontró que tanto en hombres como mujeres predominó un estilo de vida medio en el $50.0 \%$. El $37.1 \%$ de los hombres y $32.1 \%$ de las mujeres experimentan un estilo de vida alto, mientras que el $12.9 \%$ de hombres y $17.9 \%$ de mujeres lleva un estilo bajo de vida según los resultados del cuestionario PEPS-I. Al separar las calificaciones por rotación se observó el predominio de un estilo de vida alto $52.6 \%$ de los internos cursando Ginecología y en el $42.9 \%$ de los que cursaban por Pediatría al momento del estudio.

(Ver Gráfico No. 1).

\section{Gráfico No. 1: Estilo de Vida de los Internos por Rotación según Puntaje PEPS-I.}

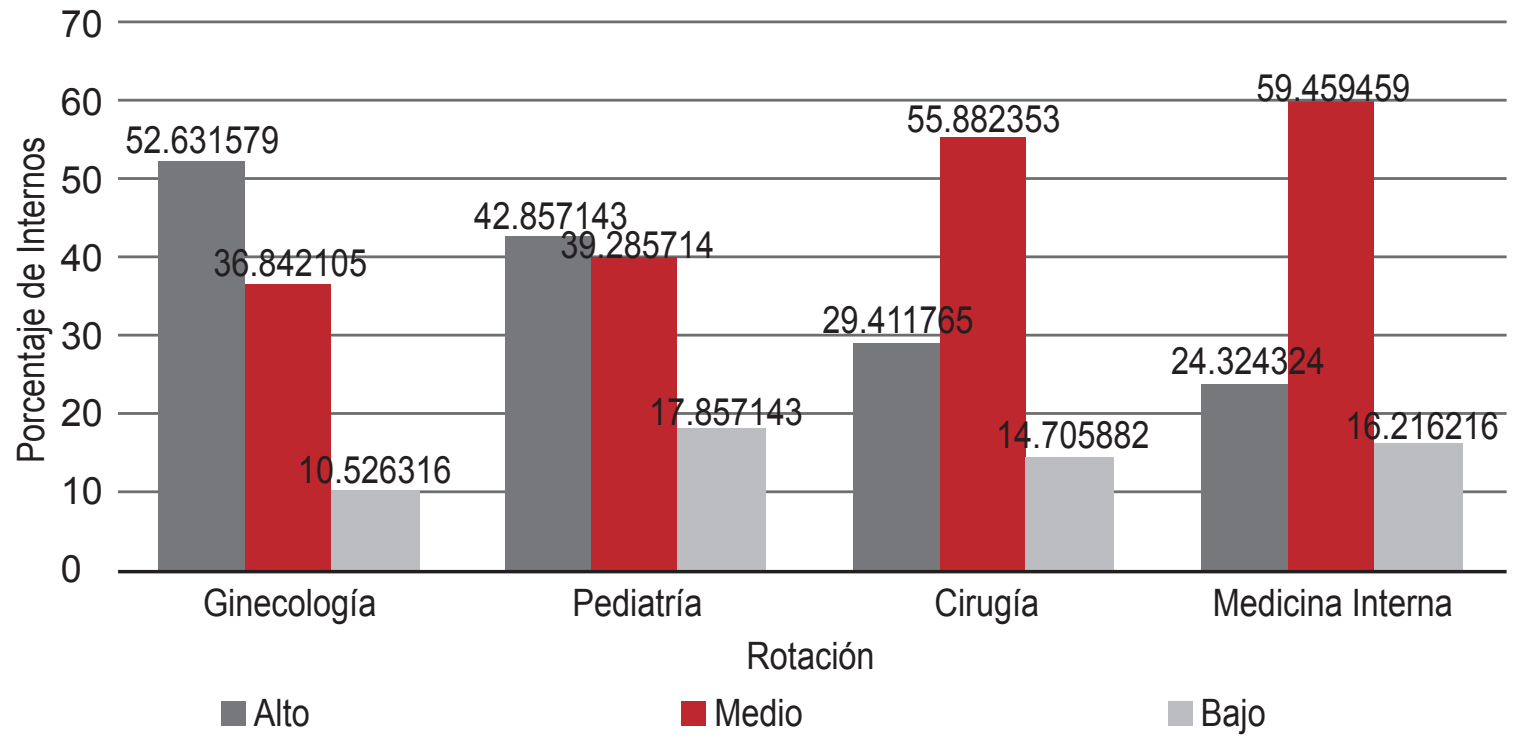

Fuente: Cuestionario de Perfil de Estilo de Vida (PEPS-I) creado por Nola Pender.

Alto

\section{DISCUSIÓN}

Sánchez-Ojeda y Luna-Bertos encontraron que las mujeres universitarias tenían prácticas más saludables en cuanto a la alimentación, ${ }^{(3)}$ sin embargo, nuestro estudio no demostró diferencias entre sexos para la categoría de nutrición. Resultados obtenidos por Rizo, Gonzales y Cortés muestran que los estudiantes universitarios de Ciencias de la Salud no aplican en sus hábitos nutricionales los conocimientos que están adquiriendo en sus estudios universitarios, manteniendo ingestas desequilibradas de macronutrientes e importantes déficits nutriciona- 
les de micronutrientes. ${ }^{(10)}$ Nuestro estudio muestra hábitos alimenticios de regular calidad en los médicos internos, pero dada la naturaleza del mismo no se obtuvieron mediciones de macro y micronutrientes ingeridos.

La dimensión más descuidada en ambos sexos fue el ejercicio, obteniendo una media de 7.02 en mujeres y 8.49 en hombres, en donde el puntaje máximo posible es de 20 puntos. Sánchez-Ojeda y Luna-Bertos obtuvieron resultados similares, estableciendo que el ejercicio físico es una de las debilidades encontradas en la población universitaria, siendo los hombres los que más ejercicio físico realizan frente a las mujeres. ${ }^{(3)}$ Las mujeres mostraron un porcentaje mínimo sobre los hombres en las áreas de responsabilidad en salud y soporte interpersonal. Estudios por De Souza y colaboradores ${ }^{(18)}$ mostraron que las personas que cuentan con redes sociales de apoyo y amistades satisfactorias (equiparables con la dimensión de soporte interpersonal) pueden aumentar hasta en un $22 \%$ sus posibilidades de alcanzar la longevidad. Otro dato interesante es el hecho de que, tener más y mejores amigos presentó ser un factor protector para la salud cardiovascular de hombres mayores, en comparación con aquellos que eran más solitarios. ${ }^{(18)}$

Según el estudio realizado por Morales, se espera que la inteligencia emocional intrapersonal se asocie con mayor capacidad de gestión del estrés; mientras que la inteligencia emocional interpersonal esté vinculada con mayor empleo de estrategias de búsqueda de apoyo. ${ }^{(19)}$ Esto manifiesta que los médicos internos poseen ambos tipos de inteligencia emocional, ya que obtuvieron puntajes aceptables en la categorías de manejo del estres y soporte interpersonal.

Los médicos internos mostraron el mejor rendimiento en la categoría de autoactualización, donde tanto hombres como mujeres obtuvieron una puntuación media de 45 de un máximo posible de 52. Varios estudios señalan que los estudiantes con mayor empleo de las estrategías productivas de afrontamiento (solución activa, búsqueda de información y guía, y actitud positiva) tienen mayor puntuación en esta dimensión. ${ }^{(13,20,21)}$ Además, los resultados obtenidos por Ortiz-Acosta y Beltrán-Jiménez nos indican que, cuando se incrementa la claridad y reparación de emociones disminuyen el agotamiento emocional y la despersonalización, aumenta la realización personal y mejora las habilidades clínicas del médico pasante de servicio social. ${ }^{(22)}$

Al igual que este estudio, Barragán-Ledesma y colaboradores no encontraron diferencias estadísticamente significativas en la distribución del estilo de vida en función de género al estudiar estudiantes universitarios del área de la salud en México. ${ }^{(23)}$ Los hombres lograron un mayor desempeño que las mujeres en las dimensiones de ejercicio y manejo del estrés, aunque la diferencia en puntaje fue mínima.

En el estudio realizado por Reyes Ticas y colaboradores, se encontró una alta prevalencia de síndrome de "burnout" (87\%) en practicantes internos del Hospital Escuela, el cual es un serio problema de salud entre la población médica que se asocia a una mala calidad de vida, bajo rendimiento y deterioro en el cuidado de pacientes. ${ }^{(16)}$

En éste estudio se observó una mejoría marcada en el estilo de vida para aquellos estudiantes de pediatría y ginecología, las cuales cuentan con programas de posgrado en el HMCR, lo que podría aminorar la cantidad de labores exigidas a los médicos internos y esto explica la tendencia a una mejor calidad de vida en estas rotaciones, contrario a las rotaciones de cirugía y medicina interna, que no cuentan con posgrado y en las cuales predomina un estilo de vida medio en más del $55 \%$ de sus internos.

\section{Conclusiones}

De manera global una tercera parte de la población resultó llevar un estilo de vida alto, mientras que la mitad llevó un estilo de vida 
medio y el restante bajo. La dimensión más débil en el estilo de vida de todo el internado, para ambos sexos fue el ejercicio físico.

El género no influyó sobre el estilo de vida de los médicos internos, tanto hombres como mujeres obtuvieron puntajes similares en el cuestionario empleado. No obstante, la rotación cursada sí presentó diferencias, las cuales resultaron más marcadas entre ginecología y medicina interna. En ginecología como pediatría (que cuentan ambas con médicos en posgrado) predominaron internos con un estilo de vida alto, mientras que en cirugía y medicina interna la mayoría lleva un estilo de vida medio.

\section{Recomendaciones}

Es necesario facilitar y promover el ejercicio físico asignando tiempo y áreas específicas para este fin dentro del predio hospitalario, $\mathrm{O}$ en su defecto, creando alianzas con gimna- sios en los alrededores del HMCR, que brinden tarifas flexibles para esta población.

Recomendamos revisar el plan de estudios de la Carrera de Medicina y asegurar que este permita llevar un estilo de vida saludable, así como extender las investigaciones sobre estilo de vida saludable a los médicos cursando el posgrado de Pediatría y Gineco-Obstetricia del HMCR.

\section{Conflicto de Interés}

No existió conflicto de interés con ninguna organización o institución. La investigación fue voluntaria, sin fines de lucro y sin financiamiento público o privado que pueda influenciar los resultados a desviarse de la realidad. Se utilizó un instrumento objetivo para poder analizarlo con imparcialidad y se ingresaron los resultados en la base de datos al azar para evitar sesgos durante la tabulación.

\section{REFERENCIAS BIBLIOGRÁFICAS}

1. Organización Mundial de la Salud. Constitución de la OMS: principios. [Internet]. Ginebra: OMS; 1946 [Consultado el 8 de mayo del 2018].

Disponible en: http://www.who.int/about/ mission/es /.

2. Sosa Hernandez AK, Barragán Ledesma LE, Linares Olivas OL, Estrada Martinez S, Quintanar Escorza MA. Análisis de la relación entre el estilo de vida y la inteligencia emocional en estudiantes universitarios. Cienc Humanismo Salud [Internet]. 2016 [Consultado el 8 de mayo del 2018];3(1):14-23. Disponible en: http://revista.medicina.uady.mx/re vista/index.php/cienciayhumanismo/ar ticle/view/47.

3. Sánchez-Ojeda MA, De Luna-Bertos E. Hábitos de vida saludable en la población universitaria. Nutr Hosp [Internet].
2015 [Consultado el 11 de mayo del 2018];31(5):1910-1919.

Disponible en: http://www.aulamedica. es/nh/pdf/ 8608.pdf.

4. Campo Y, Pombo LM, Teherán AA. Estilos de vida saludable y conductas de riesgo en estudiantes de medicina. Rev Univ Ind Santander [Internet]. 2016 [Consultado el 8 de mayo del 2018];48(3):301-309. Disponible en: http://www.scielo.org.co/pdf/suis/v48 n3/v48n3a05.pdf.

5. Farhud DD. Impact of lifestyle on health. Iran J Public Health [Intertnet]. 2015 [Consultado el 11 de mayo del 2018];44(11);1442-1444.

Disponible en: https://www.ncbi.nlm.nih. gov/pmc/arti cles/PMC4703222/pdf/I JPH-44 -1442.pdf. 
6. Atoche Pacherres CA. Desempeño laboral y estilo de vida. Caso: empresas de servicio en la ciudad de Piura-Perú, año 2015. Conference Proceedings UTMACH [Internet]. 2017 [Consultado el 11 de mayo del 2018];1(1):295-305. Disponible en: http://investigacion.utma chala.edu.ec/proceedings/index. php/utmach/article/view/126.

7. Kushner R, Mechanick J. The importance of healthy living and defining lifestyle medicine. In: Mechanick J, Kushner R. Lifestyle Medicine: a manual for clinical practice. Switzerland: Springer; 2016. p.9-15.

8. Organización Mundial de la Salud. 9. ${ }^{a}$ Conferencia Mundial de Promoción de la Salud - recursos [Internet]. Ginebra: OMS; 2016 [ Consultado el 8 de mayo del 2018]. Disponible en: http://www. who.int/healthpromotion/conferences/ 9 gchp/resources/es/.

9. Laguado Jaimes E, Gómez Díaz MP. Estilos de vida saludable en estudiantes de enfermería en la Universidad Cooperativa de Colombia. Hacia Promoc Salud [Internet]. 2014 [Consultado el 8 de mayo del 2018];19(1):68-83.

Disponible en: http://www.scielo.org. co/pdf/hpsal/v19n1/v19n1a06.pdf.

10. Rizo-Baeza MM, González-Brauer NG, Cortés E. Calidad de la dieta y estilos de vida en estudiantes de ciencias de la salud. Nutr Hosp [Internet]. 2014 [Consultado el 11 de mayo del 2018]; 29(1):153-157. Disponible en: http:// scielo.isciii.es/pdf/nh/v29n1/20original 14.pdf.

11. Secretaría de Salud (HN). Guía de estilos de vida saludables en el ámbito laboral. [Internet]. Tegucigalpa: Secretaría de Salud; 2016. [Consultado el 8 de mayo del 2018]. Disponible en: https:// bit.ly/2DJ5Fqg.
12. Jiménez MG, Martínez $P$, Miró E, Sánchez Al. Bienestar psicológico y hábitos saludables: ¿están asociados a la práctica de ejercicio físico?. Int J Clin Health Psychol [Internet]. 2008 [Consultado el 11 de mayo del 2018];8(1):185-202. Disponible en: http://www.redalyc.org/ articulo.oa?id=33780113.

13. Honduras. Congreso Nacional. Decreto Ejecutivo Número PCM-030-2015. Diario Oficial La Gaceta. 2015 Junio 24; sección A, Acuerdos y Leyes.

14. Hernández-Vargas $\mathrm{Cl}$, Dickinson-Bannack ME. Importancia de la inteligencia emocional en Medicina. Inv Ed Med [Internet]. 2014 [Consultado el 11 de mayo del 2018]:;3(11):155-160.

Disponible en: https://bit.ly/2Qxvtl4.

15. Calsin Quinto D, Quispe-Ticona IL. Relación entre estilo de vida y estrés laboral del personal de enfermería. Investig Andina [Internet]. 2013 [Consultado el 11 de mayo del 2018];12(2):13-18. Disponible en: https: //revistas.uancv.edu.pe/index.php/RCIA /article/view/ 202.

16. Reyes Ticas A, Medina MT, Mesa $X$, Paredes Y, Barahona Y, Sierra M. Estudio de síndrome de "burnout", depresión y factores asociados en los practicantes internos del Hospital Escuela. Rev Fac Cienc Med. 2012;9(1):14-20.

17. Mamani Cañazaca CE. Factores laborales y estilos de vida del personal de salud del puesto de salud Santa María, Juliaca 2015. [Internet]. Juliaca: Escuela Profesional de Enfermería, Universidad Peruana Unión; 2015. [Consultado el 17 de Junio del 2018]. Disponible en: https://bit.ly/2LZxdJJ.

18. Henrique De Souza MA, Ferreira Porto E, De Souza EL, Da Silva KI. Perfil do 
estilo de vida de longevos. Rev Bras Geriatr Gerontol [Internet]. 2016 [Consultado el 11 de mayo del 2018];19(5):819-826. Disponible en: https://bit.ly/2M2YHOg..

19. Morales Rodriguez FM. Relationships between coping with daily stress, self-concept, social skills and emotional intelligence. Eur J Educ Psychol [Internet]. 2017 [Consultado el 11 de mayo del 2018];10(2):41-8. Disponible en: https://bit.ly/2Rgm90y..

20. Wang Y, Xie G, Cui X. Effects of emotional intelligence and self-leadership on students coping with stress. Soc Behav Pers [Internet 2016 [Consultado el 11 de Mayo del 2018];44(5):853-864.

Disponible en: https://bit.ly/2H1B0Hi.

21. Azpiazu L, Esnaola I, Sarasa M. Capacidad predictiva del apoyo social en la inteligencia emocional de adolescentes
Eur J Educ Psychol [Internet]. 2015 [Consultado el 11 de Mayo del 2018];8(1):23-9.

Disponible en: http://www.redalyc.org/ articulo.oa? id=129343965003.

22. Ortiz-Acosta R, Beltrán-Jiménez BE. Habilidades clínicas, inteligencia emocional percibida y desgaste laboral en médicos pasantes de servicio social. Inv Ed Med.[Internet] 2017 [Consultado el 11 de mayo del 2018].

Disponible en: https://bit.ly/2ADI6xf.

23. Barragán-Ledesma L, González-Preza $M$, Estrada-Martinez S, Hernández-Cosain Y, Hernández-Cosain E, Ríos-Valles $\mathrm{J}$, et al. Estilo de vida y dimensiones, en estudiantes universitarios de área de la salud. Cienc Humanismo Salud [Internet]. 2015 [Consultado el 8 de mayo del 2018];2(2):53-63.

Disponible en: https://bit.ly/2ACRiRg. 